BJS215_L_Pst.nobilata_Karanja_2013

BJS216_L_Pst.variicollis* Runnymede\#2 2013 BJS217 L'Pst.variicollis* ${ }^{-}$Runnymede\#2 2013

BJS218 - L Pst.variicollis* ${ }^{*}$ Runnymede\#2 2013

BJS219_L_Pst.variicollis* Runnymede\#2_2013

BJS220_L_Pst.variicollis*_Runnymede\#2_2013

BJS221_L_Pst.variicollis* ${ }^{*}$ Runnymede\#2_2013

BJS379 P Pst.variicollis* TheLea 2014

- BJS381 P Pst variicollis* TheLea-2014

- BJS383 P-Pst.variicollis* ${ }^{*}$ TheLea 2014

BJS384_P_Pst.variicollis** TheLea_2014

BJS386_L_Pst.variicollis* ${ }^{*}$-TheLea_2014

BJS387 P Pst.variicollis ${ }^{*}$ The Lea 2014

- BJS388_L_Pst.variicollis* Runnymede\#1 2014

[ BJS564_P_Pst.variicollis ${ }^{*}$ Runnymede\#1_2015

BJS566_P_Pst.variicollis ${ }^{*}$ Runnymede\#1_2015

[ BJS403_-Pst.selmani_Moina_2011

- BJS404 Pst.selman Moina 2011

[ BJS405_L_Pst.selmani_Moina_2011

BJS407_L_Pst.selmani-Moina_2011

BJS408_L_Pst.selmani_Moina_2011

BJS377 P-Pst.variicollis* TheLea_2014

BJS378_P_Pst.variicollis ${ }^{*}$-TheLea_2014

BJS385_L_Pst.variicollis* ${ }^{*}$-TheLea_2014

- BJS501_A_Pst.variicollis* ${ }^{*}$ TheLea_2014 $^{-}$

[BJS214_L_Pst.nobilata_Karanja_2013

[ BJS380-P Pst.variicollis* TheLea 2014

4 BJS226_L_P.charybdis_Moina_2013

1 BJS199 A P. charybdis TheLea 2012

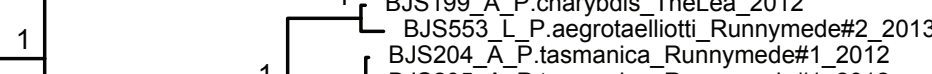

BJS205_A_P.tasmnaica_Runnymede\#1_2012

BJS239_A_P.tasmanica_Runnymede\#1_2012

BJS240_L_P.tasmanica_Runnymede\#1_2013

1 BJS241 A P tasmanica Runnymede\#12012

BJS243_L_P.tasmanica_Runnymede\#1_2012

BJS389_A_P.tasmanica_Runnymede\#1_2012

BJS397_L_P.tasmanica_Runnymede\#1_2013

BJS399_L_P.tasmanica_Runnymede\#1_2013

[ BJS554_L_P.tasmanica_TheLea_2015
4 BJS406_L_Pst.selmani_Moina_2011

Clade A

Eadya n.sp.1

Clade B

Eadya n.sp.2

Clade C

Eadya paropsidis

0.55

1

$0 . \overline{008}$

0.62 BJS245_A Unknown_Moina 2013 BJS376-P_Pst.bimacūlata Moina_2014 BJS213- - Pst nobilitata Karanja 2013 - BJS224 L_P.chaybdis_Ellendale-2013 BJS184_L_P.charybdis_Ellendale_2012

BJ Ellendale 2012

BJ 225 A.chaybdis Ellendale 2013

BJS175 [_Pst.agricola_Moina_2012

BJS17 _L_Pst.agricola_Moina_2012

BJS179_- Pst.agricola_Moina_2012

BJS10_- _st.agricola_Moina_2012

BJS183-L-Pst.agricola_Moina-2012

BJS189-Pstagricola-Moina-2012

BJS191-A-Pstagricola-Moina-2012

BJS192- P charybdis Elenda 2012

BJS194-L-Pst.agricola-Ellendale-2012

BJS202 A Unknown Moina 2012

BJS203 A Unknown Moina 2012

BJS227 L Pst.agricoTa Moiña 2013

BJS228-L_Pst.agricola-Moina-2013

BJS229 L_Pst.agricola_Moina 2013

BJS230-L-Pst.agricola-Moina_2013

BJS231_L-Pst.agricola-Moina-2013

BJS232_LPst.agicola_Moina_2013

B 2013

BJS236- Pst agricola-Runnymede\#2-2013

BJS237- - Pst.agricola-Runnymede\#2-2013

BJS238- - Pstagricola-Runnymede\#2-2013

BJS246 A Unknown Moina 2013

BJS247 A Unknown-Moina-2013

BJS248-A-Unknown- Moina-2013

BJS249-A-Unknown-Moina-2013

BJS250 A Unknown-Moina-2013

BJS251-A-Unknown-Moina-2013

BJS252_A Unknown_Moina 2013

BJS253_A Unknown Moina 2013

BJS254_A_Unknown_Moina_2013

BJS255_A Unknown-Moina_2013

BJS256_A_Unknown_Moina_2013

(3)

JJS250-A-Unknown- Mina-2013

JJS260-A Unknown-Mina_2013

BJS261-A-Unknown-Mina-2013

BJS262-A-Unknown-Moina-2013

BJS263-A-Unknown-Moina-2013

BJS264-A -Unknown-Moina-2013

BJS265-A-Unknown-Moina-2013

BJS266 A Unknown Moina 2013

BJS267-A-Unknown-Moina-2013

BJS268_A Unknown Moina 2013

BJS269_A Unknown Moina 2013

BJS287_A Unknown_Moina_2014

BJS288_A_Unknown-Moina_2014

BJ 289-Ankown_Moina_2014

BJS290_A_Unknown_Moina_2014

BJS292-A-Unknown-Mina-2014

BJS294-A-Unknown-Runnymede\#1 2014

-

BJS296-A-Unknown-Runnymede\#1-2014

BJS297-A Unknown-Runnymede\#1-2014

BJS298-A Unknown-Runnymede\#1-2014

BJS299-A - Unknown-Runnymede\#1-2014

BJS300 A Unknown-Runnymede\#1-2014

BJS301_A_Unknown_Runnymede\#1-2014

BJS302_A_Unknown_Runnymede\#1-2014

BJS304-A-Unknown-Runnymede\#1-2014

BJS305-A-Unknown-Runnymede\#1-2014

BJS307-A-Unknown-Runnymede\#1-2014

BJS308-A-Unknown-Runnymede\#1-2014

BJS309-A-Unknown-Runnymede\#1-2014

J 312-A Unknown-Runnymede

BJ

BJS314-A Unknown-Runnymede\#1-2014

BJS315-A Unknown-Ellendale 2014

BJS316-A-Unknown-Ellendale-2014

BJS317-A-Unknown-Ellendale-2014

BJS318-A-Unknown-Ellendale-2014

BJS319-A_Unkown_Moina_2014

BJS320_A-Unkown_Moina-2014

BJS321-A-Unkown_Moina_2014

BJS322-A-Unkown-Moina_2014

BJS323-A-Unkown-Moina-2014

BJS324_A_Pst.agricola_Ellēndale_2014

B

BJS326_A_Pst.agricola_Ellendale_2014

BJS328-A-Pstagricola-Ellendal-2014

BJS320-A-Pst agricol-E -

BJS330-A Pst agricola-Ellendale-2014

BJS331-A-Pst.agricola-Ellendale-2014

BJS332-A-Pstagricola-Ellendale-2014

BJS333-A-Pst.agricola ${ }^{-}$Ellendale ${ }^{-2014}$

BJS334-A Pst.agricola-Ellendale ${ }^{-2014}$

BJS335_A Pst.agricola Moina 2014

\title{
BJS336_A Pst.agricola_Moina_2014
}

BJS337_A_Pst.agricola_Runnymede\#1_2014

BJS338_A_Pst.agricola_Runnymede\#1-2014

BJS339_A Pst.agricola_Runnymede\#1_2014 BJS341__Pst.agricola_Runnymede\#1_2014 BJS34- - Pst.agricola-Runnymede\#1-2014 BJS346--Pstagricol-Runnymede\#1-2014 BJS349-_Pstagricol-Runnymedet1-2014 BJS 347- - Pst.agricola-Runnymede\#1-2014 BJS350-L-Pst.agricola-Ellendale 2014 BJS348 L Pstagricola Runnymede\#1 2014 BJS351- L-Pst.agricola-Ellendale 2014 BJS352- L-Pst.agricola-Ellendale ${ }^{-2014}$ BJS353-L-Pst.agricola Ellendale ${ }^{-2014}$ BJS354 L Pst.agricola Ellendale 2014 BJS355_L_Pst.agricola_Ellendale- 2014 BJS359_L_Pst.agricola_Moina 2014 BJS361_L-Pst.agricola_Moina_2014 BJS363_L_Pst.agricola-Moina-2014 BJS364_L_Pst.agricola_Moina_2014 JJ367-Pst_ogricola-Moina-2014 BJS368-_Ps.agricola_-Moina-2014 BJS369-P-Pst agricola-Moina-2014 BJS370-P Pst.agricola-Moina-2014

Clade D2

Eadya n.sp.3 